\title{
TRAS LAS HUELLAS DE SANTOS VITO VÉLIZ
}

\author{
Nohemy Lizeth Rivera Gutiérrez ${ }^{1, *}$ \\ ${ }^{1}$ Universidad Nacional Autónoma de Honduras
}

Recibido: 08/noviembre/2020

Aceptado: 08/diciembre/2020

DOI: https://doi.org/10.5377/ce.v13i2.11519

\begin{abstract}
RESUMEN
El artículo plasma una breve reseña de la trayectoria profesional y académica del arqueólogo hondureño Santos Vito Veliz Ramírez, abordando su formación académica, experiencia profesional, investigaciones, publicaciones y con énfasis en su labor universitaria. Se realizó una recopilación de sus obras, publicaciones, documentos administrativos que dan cuenta de su trayectoria y entrevistas a personas que se relacionaron con el profesor Veliz, tanto como docente y profesional. Su vida ha dejado un extenso legado a la arqueología y arqueoastronomía de Honduras.
\end{abstract}

Palabras clave: Santos Vito Veliz, arqueología, arqueoastronomía, Honduras.

\begin{abstract}
The article presents a brief review of the professional and academic career of the Honduran archaeologist Santos Vito Veliz Ramírez, addressing his academic training, professional experience, research, publications and with emphasis on his university work. It was made a compilation of his books, publications, administrative documents that give an account of his career and interviews with people who were related to Professor Veliz, both as a teacher and as a professional. His life has left an extensive legacy to the archeology and archaeoastronomy of Honduras.
\end{abstract}

Keywords: Santos Vito Veliz, archaeology, archaeoastronomy, Honduras.

\section{Introducción}

El profesor Santos Vito Veliz nació el 15 de junio de 1939, en Esquipulas del Norte, Olancho. Formó una familia con la señora Edith de Veliz y sus cinco hijos, y posteriormente sus nietos. Una vida dedica a la arqueología, la arqueoastronomía y el patrimonio cultural. Muere el 8 de julio de 2020.

\section{Sus estudios}

Sus primeros años de estudio los cursó en Esquipulas del Norte en la escuela "Angel G. Hernández"; y en Olanchito, Yoro, en la escuela "San Jorge" y escuela "Modesto Chacón", entre 1948 a 1955. Realizó sus estudios secundarios en el St. John's College en Belice, en los años de 1956 a 1960, con el apoyo de una beca de los Padres Jesuitas (Compañia de Jesús).

*nohemy.rivera@unah.edu.hn (D) https://orcid.org/0000-0003-2317-8285 
Posteriormente, de 1960 a 1963, realizó sus estudios universitarios en St. Louis University en St. Louis, Missouri, Estados Unidos de América; de 1963 a 1964 estudió en el Instituto de Literatura, Puente Grande, Jalisco, México; y obtuvo su Licenciatura (B.A.) en Filosofía y Letras en St. Louis University, St. Louis, Missouri, Estados Unidos de América de 1964 a 1966. Estos estudios fueron realizados mediante una beca de la Compañía de Jesús.

Con una beca de la Universidad Nacional Autónoma de Honduras (UNAH), siguió con estudios de Posgrado en la Universidad de Kansas en Lawrence, Kansas, obteniendo una Maestría (M.A.) en Antropología, con especialidad en Arqueología, de 1969 a 1972. Su tesis se llamó "Análisis Arqueológico de la Cerámica de Piedra Blanca, Noreste de Honduras", enfocado al análisis descriptivo de una colección de tiestos de barro actualmente en el Museo Peabody de la Universidad de Harvard

Entre 1988 y 1989, durante un año estuvo como Profesor Visitante en la Universidad de Texas en Austin, Texas, E.U.A., donde estudió la escritura, el arte y la religión maya, junto a la Dra. Linda Schele. Esta oportunidad se materializó a través de una beca Fullbright del Gobierno de los Estados Unidos. En ese período tuvo la oportunidad de participar en diferentes conferencias y eventos, principalmente de epigrafía:

- Taller de Escritura Maya en la Universidad de Texas, Austin, Texas, con la Dra. Linda Schele, en marzo 1988.

- Taller de Escritura Maya en la Universidad de Pennsylvania, Philadelphia, con Lic. David Stuart y otros, en abril 1988.

- Reunión de la Sociedad para la Arqueología Americana, Phoenix Arizona, abril 1988.

- Taller de Escritura Maya en la Universidad Suroeste Estatal de Texas en San Marcos, Texas, impartido por Nicholas Hopkins y Katherine Josserand, en octubre 1988.

- Seminario sobre Teotihuacán en Dumbarton Oaks, Washington, D.C., en octubre 1988.

- Taller de Escritura Maya en la Universidad de Texas, Austin, Texas, con la Dra. Linda Schele, marzo 1989.

A partir de una beca patrocinada por UNESCO, participa en el Seminario Internacional sobre Parques Nacionales y Reservas Equivalentes en Canadá, Hawái y los Estados Unidos, con lo cual recorrió los principales parques culturales en el Suroeste de los Estados Unidos (1983). Adicionalmente, con otra beca de la UNESCO participa en el recorrido de las principales ciudades mayas en Guatemala, Belice y México (1987).

Su lengua materna fue el español, pero dada su experiencia educativa y laboral tuvo como segunda lengua el inglés; así como aprendió elementos esenciales del francés, latín y griego.

\section{Su carrera profesional}

La experiencia laboral del profesor Vito Veliz fue principalmente en el sector público, asimismo en instituciones de educación superior como profesor universitario. Sin embargo, su labor fue mucho más amplia. Su primer trabajo se remonta a 1966, después de terminar sus estudios de licenciatura, laboró como docente en el Colegio San José de la ciudad de El Progreso, en la zona norte de Honduras. Posteriormente, durante dos años fue administrador de producción en la fábrica de telas Textiles Rio Lindo, en Comayagüela, Honduras. 
Después de regresar de sus estudios de posgrado, fue docente en varias universidades, como en la Universidad José Cecilio del Valle, la Universidad Pedagógica Nacional Francisco Morazán (UPNFM) y la Universidad Nacional Autónoma de Honduras. De 1973 a 1991, impartió cursos generales de Antropología y de Arqueología en distintas ocasiones en la otrora Escuela Superior del Profesorado, en la Universidad Nacional Pedagógica Francisco Morazán y la Universidad José Cecilio del Valle.

Con el Instituto Hondureño de Antropología e Historia (IHAH), de 1975 a 1991 fue jefe del Departamento de Investigaciones Antropológicas. Allí mismo, creo los Estudios Antropológicos e Históricos del IHAH (1978), realizó actividades administrativas en el Proyecto Arqueológico Copán (1981 a 1986), y llegó a ejercer el máximo cargo de esa institución, fungiendo como Gerente de la institución desde noviembre 1986 a marzo 1987.

En el año 1981, coordinó el "Primer Seminario de Arqueología Hondureña" que se celebró del 10 al 12 de junio de 1981, en el Teatro Manuel Bonilla, Tegucigalpa. Este Seminario tenía como objetivo la divulgación e intercambio entre investigadores de los proyectos arqueológicos más importantes que se estaban desarrollando en ese momento, como ser el Proyecto Arqueológico El Cajón, el Proyecto Arqueológico Sula, el Proyecto Arqueológico Copán, entre otros. El evento fue creado y organizado por el Instituto Hondureño de Antropología e Historia, y en esa ocasión contó con la colaboración de la Empresa Nacional de Energía Eléctrica, Universidad Nacional Autónoma de Honduras y la Secretaría de Cultura y Turismo (Véliz, 1981).

Desde 1969 participó en diversos proyectos arqueológicos, junto a investigadores nacionales e internacionales. Sus primeros pasos fueron en Estados Unidos, durante su posgrado, primero en los Llanos de Kansas, con las Universidades de Kansas y la Estatal de Kansas, en el verano de 1969; y en el Suroeste de los Estados Unidos, con la Universidad de Colorado, en el verano de 1970. Posteriormente, su labor arqueológica se centró a estudiar sitios arqueológicos en territorio hondureño. Los principales sitios estudiados y los equipos de trabajo son los siguientes:

- Islas de la Bahía y Trujillo en el proyecto de la Universidad de Harvard, con el Dr. Gordon Willey y el Lic. Paul Healey, de febrero a marzo 1973.

- Islas de la Bahía en el proyecto de la Universidad de Texas en Austin, con el Dr. Jeremiah Epstein, en julio 1975.

- Copán, en el proyecto de la Universidad de Harvard, con el Dr. Gordon Willey y el Lic. Richard Leventhal, de febrero a marzo 1976.

- Excavaciones en el sitio arqueológico de Currusté en el Valle de Sula, con el Lic. George Hasemann, en 1978.

- Valle de Sula, en el proyecto del Instituto de Antropología, con el Dr. John Henderson de la Universidad de Cornell, en 1978.

- Reconocimiento arqueológico preliminar de El Cajón, con el Lic. George Hasemann, de agosto a septiembre de 1979.

- Reconocimiento arqueológico de El Cajón, con el Dr. Kenneth Hirth de la Universidad de Kentucky, de enero a junio de 1980.

- Excavaciones en el Valle de Sula, en las Islas de la Bahía y en La Ceiba, de junio a septiembre de 1989. 
- Observaciones del Sol y su relación con los monumentos de Copán, con la Profa. María Cristina Pineda de Carías, en el proyecto del Observatorio Astronómico de Centroamericano de Suyapa de la Universidad Nacional Autónoma de Honduras, desde 2000 a 20012.

En Copán, su labor fue extensa, iniciando como miembro de un equipo de arqueólogos para elaborar un proyecto de investigación y restauración para Copán, por un período de cinco años desde abril de 1975. En ese mismo año, participó en el equipo de la Institución Smithsoniana para iniciar el tratamiento de la microflora que afecta los monumentos de Copán. Posteriormente, en 1978, participó en la evaluación de la primera temporada de campo del Proyecto Arqueológico Copán (PAC), convirtiéndose en supervisor del Proyecto Arqueológico Copán, durante las primeras dos temporadas de campo (1978 - 1979), cuyo director de proyecto era el Dr. Claude F. Baudez para el Musée de L'Homme de París. Ya en 1981, y hasta 1986, fue el subdirector del Proyecto Arqueológico Copán. De igual manera, fue miembro de los grupos de trabajo para las exhibiciones arqueológicas en Copán, para el Plan Interpretativo y de Educación Ambiental en Copán y para tres audiovisuales sobre la Cultura Maya (1982-1985). Y más tarde, en 1999 y en 2003, fue supervisor de proyectos arqueológicos en Copán, como consultor para el Instituto Hondureño de Antropología e Historia.

Con la Organización de Estados Americanos (OEA), participó en el equipo de trabajo para estudiar el problema del deterioro de la piedra de los monumentos de Copán, en enero 1975; también la OEA lo invitó a evaluar un programa de entrenamiento de técnicos en arqueología en Panamá en 1985; y para impartir una serie de conferencias sobre culturas prehispánicas en Mesoamérica en la Ciudad de Panamá en el año 1986.

Para la UNESCO, fue miembro del equipo de trabajo que elaboró un plan de actividades a corto, mediano y largo plazo para las Ruinas de Copán en 1975; y entre 1981 y 1982 coordinó un equipo compuesto por personal técnico del CATIE, Costa Rica; fue arqueólogo del Proyecto Arqueológico Copán, para preparar un Plan de Manejo y Desarrollo de Copán. En 1988, fue parte de un equipo de trabajo en la temática del tráfico ilícito de objetos culturales junto a arqueólogos centroamericanos y norteamericanos.

Otra de las actividades que el profesor Vito Veliz realizaba era la traducción especializada al inglés y español de documentos, trabajando en las traducciones de las publicaciones del Proyecto Arqueológico Copán, Segunda Fase, 1986-1991. Se publicaron los volúmenes I, II y III en español. Fue traductor (inglés a español) de la Guía de Casa K'inich, el Museo del Niño en Copán Ruinas y de tres artículos sobre las investigaciones arqueológicas en Copán de la Universidad de Queensland, Australia (2002-2003).

En la década de los 90 se traslada a Estados Unidos donde reside en Los Ángeles, California. Allí se desempeña en diversos trabajos, algunos asociados a su experiencia previa, como en el área docente en la enseñanza del inglés con las empresas Lexicon e Inglés sin Barreras, como editor y columnista en el periódico El Sol de América, y en traducciones y escritos diversos. Resulta relevante mencionar que el profesor Veliz relataba todas sus experiencias laborales, desde sus trabajos como distribuidor de periódicos hasta Gerente del IHAH, con el mismo detalle, y revelando el valor de cada experiencia en su vida.

A finales de esa década, regresa a Honduras, donde trabaja en la UPNFM dictando cursos generales de Antropología y seminarios sobre Etnología en la Educación a Distancia en Comayagua y Choluteca, entre los años 1999 y 2000.

También el profesor Vito Veliz trabajo entre 2004 y 2006 como asesor en algunos proyectos con la Asociación Copán para el Desarrollo Integral de Parques Arqueológicos (DIPA), con la Secretaría de Turismo y con el IHAH en el Proyecto de Desarrollo Regional en el valle de Copán (PDRVC). Con este último, fue nombrado Subgerente de Patrimonio desde febrero de 2006 a febrero de 2007. 
Tabla 1: Libros

\begin{aligned} & \hline No. Título \\ & \hline 1 Rudimentos de Antropología. Imprenta López, Tegucigalpa, 1988. \\ & 2 Copán en el Mundo Maya. Litográfica Comayagüela, 1990. \\ & 3 Mitos: Alma de Culturas. Litocom, Tegucigalpa, 2001. \\ & 4 Manual de los Monumentos de Copán. Asociación Copán (coautor y editor), 2010. \\ & Autor del capítulo: Investigaciones en la Plaza Principal. \\ & \hline\end{aligned}

Fuente: Elaboración propia a partir de la hoja de vida del profesor Vito Veliz y repositorios institucionales.

Durante su carrera, fue un divulgador de la defensa del patrimonio cultural, particularmente del patrimonio arqueológico de Honduras. Realizó innumerables charlas y seminarios a distintos grupos, en todo el país. Y durante su trayectoria, organizó innumerables Seminarios sobre Arqueología Hondureña.

\section{Investigación y Publicaciones}

El profesor Vito Veliz, durante su trayectoria profesional generó una notable producción científica, a partir de sus trabajos individuales y colaboraciones con otros investigadores. La primera publicación identificada fue un artículo en la revista Ariel en el año 1973, con el nombre Arqueología: Breves Ideas. A partir de allí, se registran libros y artículos en revistas nacionales e internacionales (tabla 1, tabla 2 y tabla 3).

Su primer libro Rudimentos de Antropología, publicado en 1988, presenta una visión general del campo antropológico, para que fuera una fuente de consulta y que motivara el interés en esta ciencia. En este libro nos invita a reflexionar en el papel de la ciencia en nuestros países y la relación de la humanidad con el planeta y entre individuos. Llama la atención a lo siguiente:

Nuestras instituciones educativas, especialmente las Universidades, deben asumir su papel en el quehacer investigativo y en la mentalidad crítica con que se debe apreciar y adoptar todo. Nuestros gobiernos y las empresas privadas deben adoptar planes de desarrollo, pero no sólo para exportar y explotar, sino también para el consumo local y para crear el mejor ambiente en que pueda desarrollarse cada ciudadano (Véliz, 1988: 83).

Esa reflexión que realizó a finales de la década 80 fue congruente con su trabajo en instituciones de educación superior y su involucramiento en la investigación científica. Y, sigue vigente su llamado a la planificación bajo esquemas de desarrollo sostenible.

En 2010, colaboró con la Asociación Copán en la edición, junto al arqueólogo Ricardo Agurcia, del Manual de los Monumentos de Copán Honduras, así como en la autoría de la sección Investigaciones en la Plaza Principal, donde se sintetiza la historia de las investigaciones arqueológicas en la Plaza Principal. Este Manual consiste en la descripción del arte y la escritura en los monumentos de la Plaza Principal, con el aporte de Peter Biro y Dorie Reents-Budet (Agurcia Fasquelle y Véliz, 2010).

Su último libro, que está en proceso de publicación, fue escrito junto a la profesora María Cristina Pineda de Carías y el arqueólogo Ricardo Agurcia Fasquelle, referente a la arqueoastronomía en Copán, uno de sus ejes de trabajo durante las últimas dos décadas. 
Como artículos, su producción fue significativa, siempre enfocado a temáticas arqueológicas, y particularmente Copán. En la década de los 70, se registran artículos en la revista Ariel; en Sectante, publicación de la Secretaría de Cultura y Turismo; y en la Revista de la Academia Hondureña de Geografía e Historia.

Tabla 2: Publicaciones nacionales e internacionales*

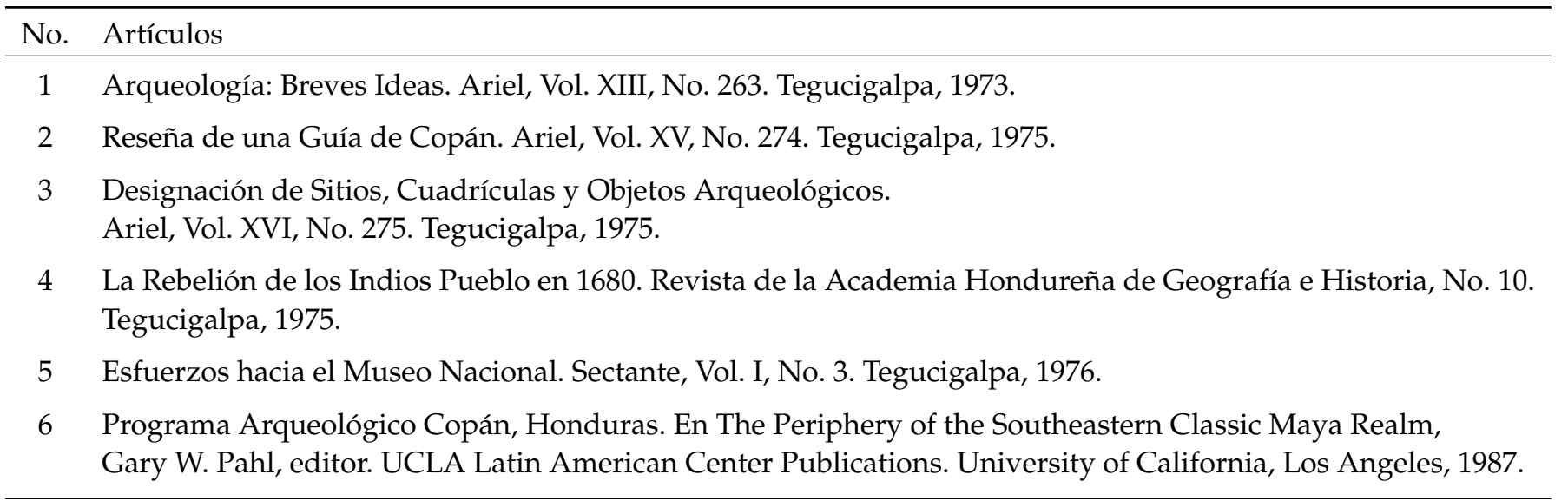

Fuente: Elaboración propia a partir de la hoja de vida del profesor Vito Veliz y repositorios institucionales.

${ }^{*}$ Los artículos en las publicaciones del IHAH y la UNAH aparecen en las tablas siguientes.

Sin embargo, su mayor producción científica quedó registrada en las publicaciones en la Revista Yaxkin y en los informes técnicos generados para el Instituto Hondureño de Antropología e Historia (tabla 3). En los inicios de la revista Yaxkin en 1975, formó parte del equipo de edición y fue el autor de los dos instructivos sobre investigaciones arqueológicas en Honduras, que aparecen en los primeros dos números de la Revista (Yaxkin Vol. I, No. 1 y Yaxkin Vol. I, No. 2).

Luego, entre 1986 a 1991, fue coordinador asistente de las publicaciones del Proyecto Arqueológico Copán, en su segunda fase. Durante ese período se publicaron en español, los volúmenes I, II y III del proyecto.

Formó parte del inicio de la Revista Yaxkin del Instituto Hondureño de Antropología e Historia, en 1975. Asimismo, redacto los Instructivos No. 1 (Yaxkin, Vol. I, No. 1) y No. 2 (Yaxkin, Vol. I, No. 2) sobre investigaciones arqueológicas en Honduras. Asimismo, publico artículos en varias ocasiones en la Revista.

Entre 1986 a 1991, fue coordinador asistente de las publicaciones del Proyecto Arqueológico Copán, en su segunda fase. Durante ese período se publicaron los volúmenes I, II y III en español

\section{Sus huellas en la Universidad Nacional Autónoma de Honduras}

La historia del profesor Vito Veliz en la UNAH inicia en la década del 70, impartiendo cursos introductorios de Antropología y Arqueología en el Departamento de Ciencias Sociales, desde 1972 hasta 1975, y posteriormente en el año 1999.

El primer registro de publicación en la UNAH fue en 1973, con el artículo "Papel del subtema político en Fuenteovejuna" en Presencia Universitaria, medio publicado por la Editorial Universitaria, que se denominaba el órgano informativo doctrinario y cultural de la UNAH, a cargo de Jubal Valerio Hernández, Roberto Sosa, Ventura Ramos y Longino Becerra. También, publicó en la Revista de la Universidad sobre la cerámica de Cuyamel con Roberto Reyes Mazzoni, en 1974 y sobre la cerámica de la Isla de Roatán en coautoría con Paul Healey y Gordon Willey en 1977 (tabla 4). 
Tabla 3: Publicaciones y proyectos arqueológicos con el IHAH

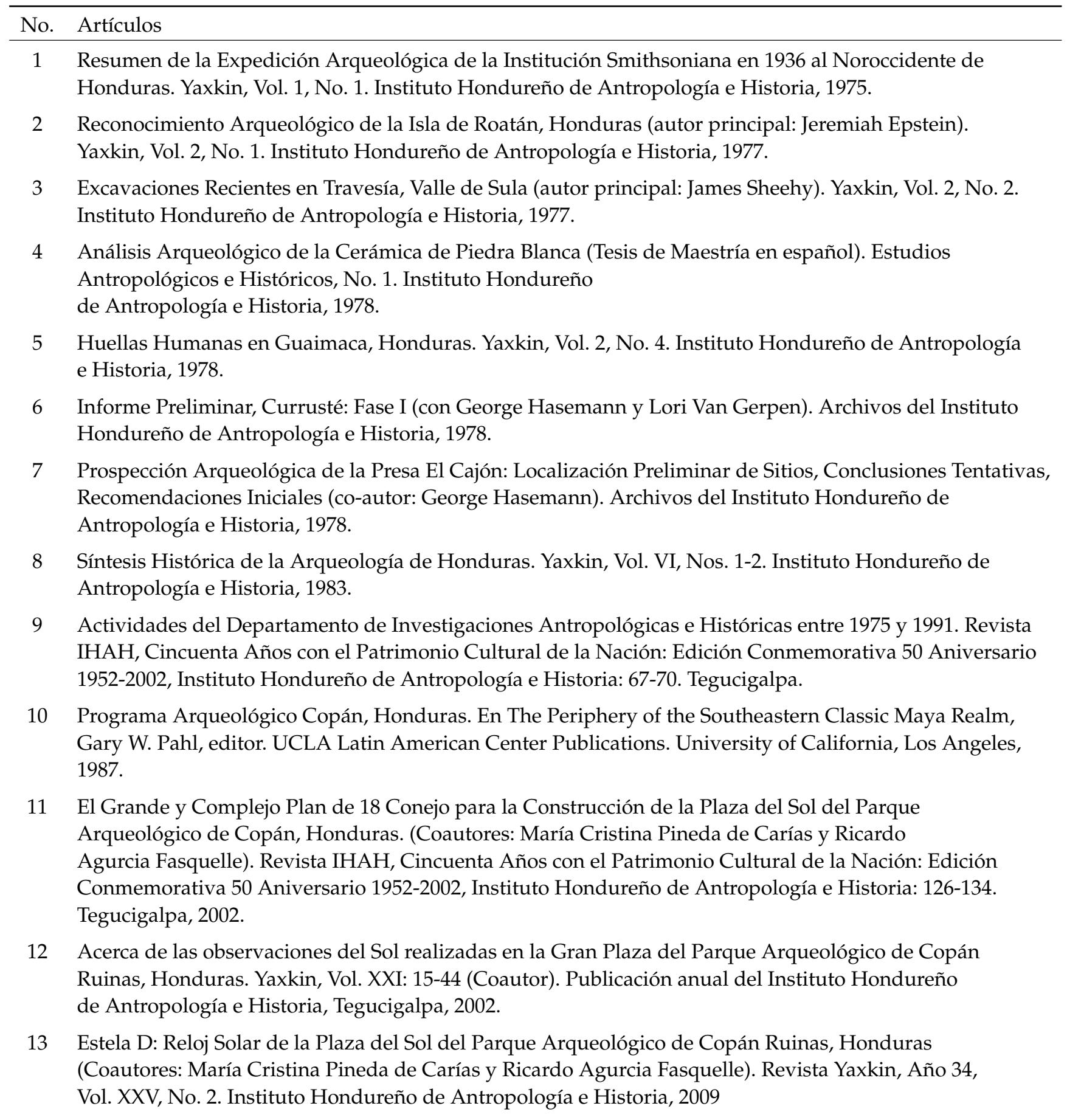

Fuente: Elaboración propia a partir de la hoja de vida del profesor Vito Veliz y repositorios institucionales. 
Tabla 4: Artículos publicados en diversas revistas de la Universidad Nacional Autónoma de Honduras, en temáticas de las ciencias sociales*

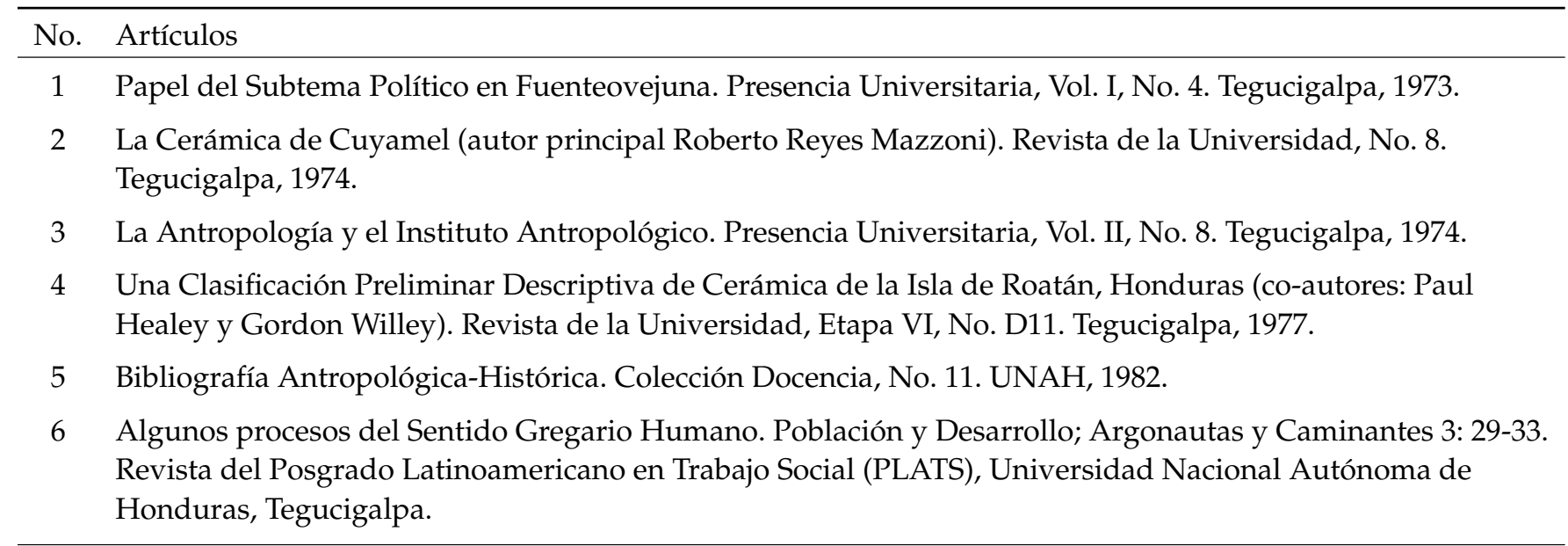

Fuente: Elaboración propia a partir de la hoja de vida del profesor Vito Veliz y repositorios institucionales.

* Los artículos relacionados con las Ciencias Espaciales aparecen en una tabla aparte.

En 1982, escribe el libro Bibliografía Antropológica-Histórica, publicado por la Editorial Universitaria de la UNAH.

\section{Sus huellas en la Facultad de Ciencias Espaciales}

Desde febrero del año 2000, trabaja en el Observatorio Astronómico Centroamericano de Suyapa (OACS/UNAH), mediante contrato temporal, y finalmente es nombrado en enero de 2002.

A partir del año 2009, el OACS se convierte en la Facultad de Ciencias Espaciales, donde el profesor Veliz jugó un papel fundamental en esta labor. En la Facultad, fue nombrado jefe del recién creado departamento de Arqueoastronomía, donde junto al equipo de profesores e instructores, Cristina Argueta, Cesar Rodríguez, Arnulfo Ramírez y Bertilio Amaya, iniciaron la conformación de este campo del conocimiento en Honduras. Bajo el liderazgo del profesor Veliz, crearon las primeras asignaturas de Arqueoastronomía como oferta académica general optativa en la UNAH. Y de forma paralela, se continuaron los proyectos de investigación, los proyectos de vinculación con la sociedad y los primeros pasos para el diseño de una carrera propia del campo del conocimiento.

Durante ese período, gestionó la creación de la asignatura general optativa "Introducción a la Arqueoastronomía" que es impartida por el Departamento de Arqueoastronomía desde el año 2008; y del "Diplomado en Arqueoastronomía Maya" impartido por el profesor Vito Veliz cuya primera edición fue en Copán Ruinas para guías turísticos y otras personas interesadas en la temática.

Desde 2008, fue docente de la asignatura "Introducción a la Arqueoastronomía". Una anécdota de esa primera clase de Arqueoastronomía que se sirvió:

Cuando hicimos el primer viaje a Copán Ruinas, con la primera promoción de la clase de Arqueoastronomía, él (profesor Veliz) era nuestro guía por el parque arqueológico y se notaba su emoción por llevar sus alumnos. Nos anduvo por el grupo principal y Las Sepulturas. Como a las cuatro de la tarde escuché una alumna decir: ay no, y es que ese señor no se cansa... Él era incansable en lo que le apasionaba (Bertilio Amaya, comunicación personal, 2020). 
En 2010, fue profesor de la asignatura Seminario de Arqueoastronomía, para la quinta promoción de la Maestría Académica Centroamericana en Astronomía y Astrofísica, de la Facultad de Ciencias Espaciales. A partir de un trabajo de investigación y gira de campo a Copán durante el paso del Sol por el cenit en agosto de 2010, los estudiantes Roberto Schöngarth, Deborah Forrest, Edward Milla y Ricardo Pastrana realizaron la publicación del artículo "Posición de la Estelas 10 y 12 respecto del Parque Arqueológico de Copán Ruinas". Asimismo, se impartieron charlas para maestros y público en general de Copán y se realizó un sondeo sobre las creencias populares relativas a los astros en la comunidad de Hacienda Grande de Copán Ruinas (Schöngarth et al., 2011) (ver figura 1).

Estas giras de trabajo generan todo tipo de anécdotas, como las relacionadas a su energía y capacidad de caminar en el campo y montañas, tanto que subía más rápido que algunos de sus estudiantes (más jóvenes que él) y otros profesores. O sobre la cantidad de gente que lo conocía en Copán (y en otras regiones de Honduras también), lo abordaban para platicar con él:

Cuando uno iba platicando con él (profesor Veliz), en las calles de Copán, ni una conversación se terminaba, porque salía alguien a ofrecerle algo... y decía: Ya los alcanzo (R. Schongarth, comunicación personal, diciembre del 2020).

También las anécdotas sobre sus relatos sobre la historia y arqueología de Copán:

Cuando contaba las viejas historias de la gente que estudiaba Copán, nosotros decíamos: pucha, así como habla de Morley, parece que hubiera estado allí (R. Schongarth, comunicación personal, diciembre del 2020).

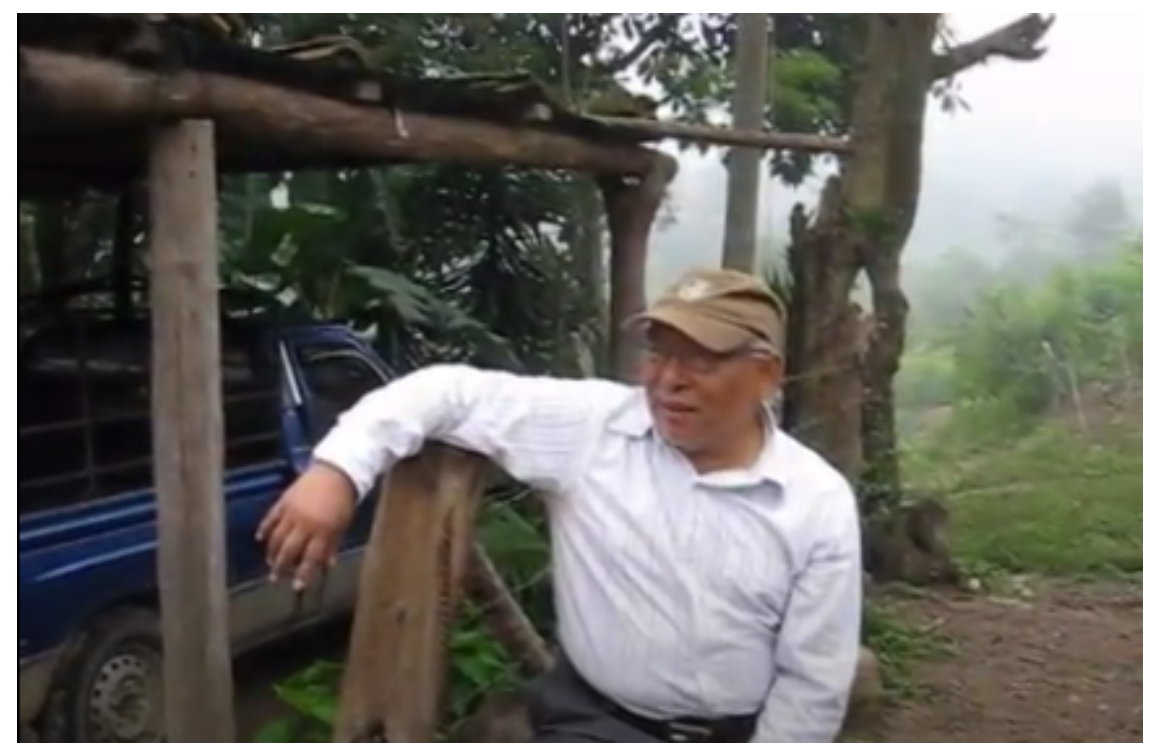

Figura 1: Entrevistas con pobladores de las comunidades de Copán durante la gira de campo con estudiantes del Seminario de Arqueoastronomía en la Maestría en Astronomía y Astrofísica. Créditos: Roberto Schöngarth.

En octubre 2009, en el marco del proyecto PCI-AECID “Desarrollo de nuevas tecnologías para la protección y preservación del patrimonio cultural y hábitats humanos en Honduras" del programa de colaboración conjunta de la Agencia Española de Cooperación Internacional y Desarrollo (AECID), realizan, junto a Rafael Corrales y Alexis Sánchez, una visita a Madrid (figuras 2 y 3). Esta actividad es organizada por la profesora María Cristina Pineda de Carías (UNAH), Francisco Burillo Mozota (Universidad de Zaragoza, España) y Juan Gregorio Rejas (Universidad Politécnica de Madrid). 

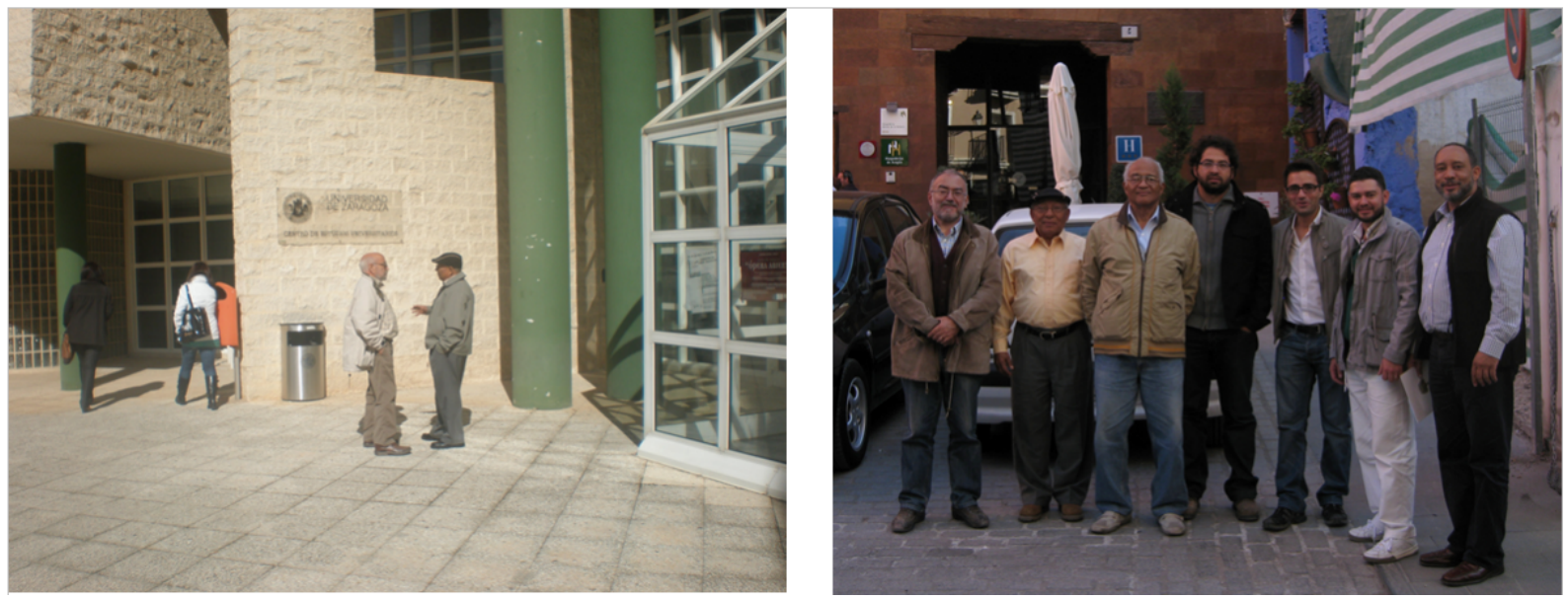

Figura 2: Visita académica a la Universidad de Zaragoza 2009. Créditos: Juan Gregorio Rejas

Durante esta visita, participó como ponente en el VIII Congreso Ibérico de Arqueometría, donde se expusieron las experiencias de trabajo en arqueoastronomía entre los equipos de Honduras y España. Asimismo, el profesor Vito Veliz participó como conferencista en el Seminario "Nuevas Tecnologías para la gestión del patrimonio en Centroamérica", en la Universidad Politécnica de Madrid. Allí impartió la conferencia "Contexto actual del patrimonio arqueológico en Honduras. Más allá de Copán". Este evento formaba parte de las actividades de difusión del proyecto PCI-AECID.

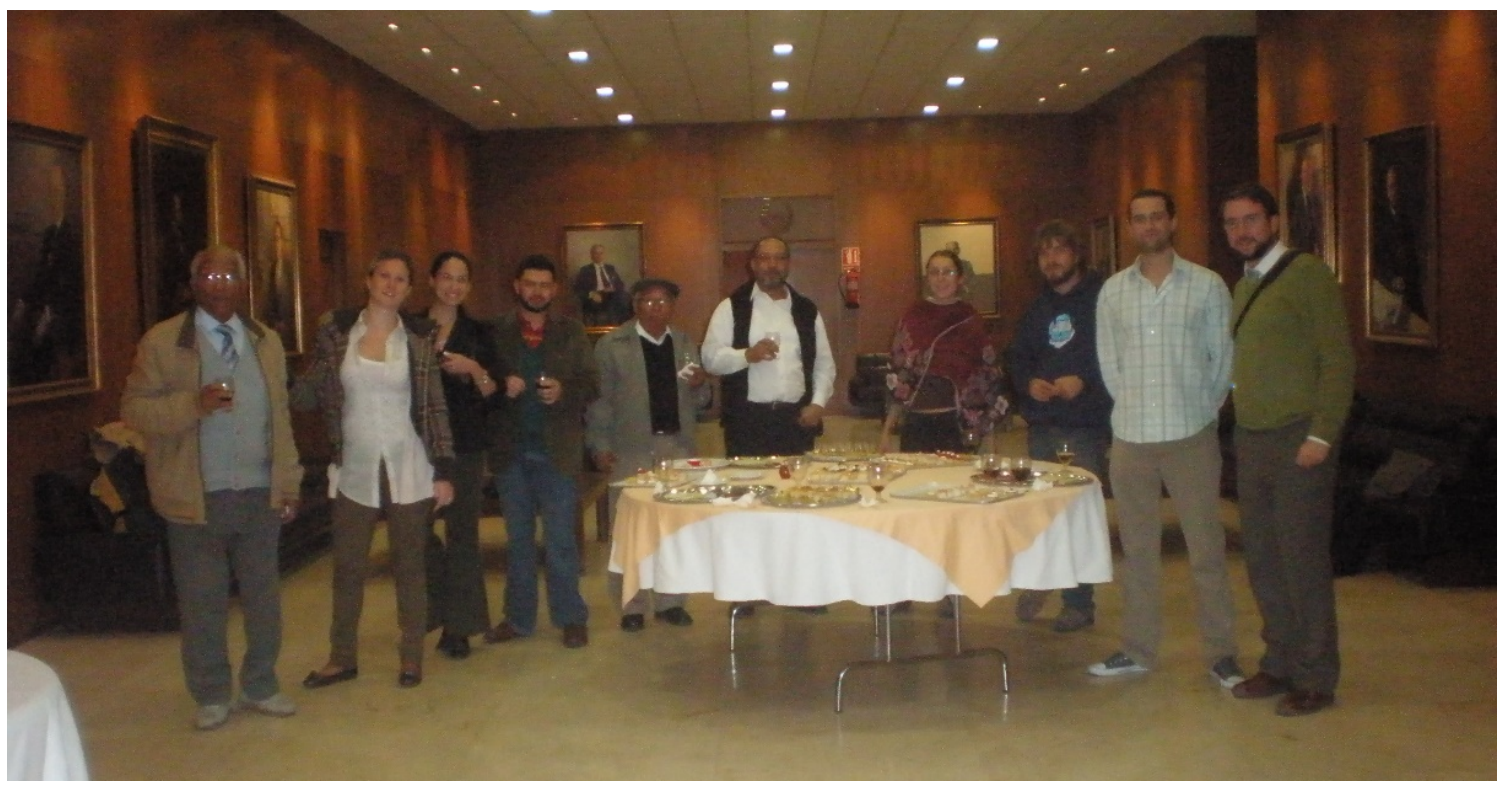

Figura 3: Clausura del Seminario en el Hall Noble de los directores de la Escuela de Caminos, UPM, Madrid 2009. Créditos: Juan Gregorio Rejas

Como parte del proyecto, el profesor Vito Veliz junto al profesor Juan Gregorio Rejas y otros investigadores, realizaron visitas de campo en Honduras (figura 4).

Dándole seguimiento a este proyecto iniciado con los investigadores españoles, se realizó el proyecto de investigaciones arqueoastronómicas en el Valle de Otoro con el apoyo financiero de la Dirección de Investigación Científica (DICU) de la UNAH (figura 5). Como resultado de este proyecto se publicó el artículo 

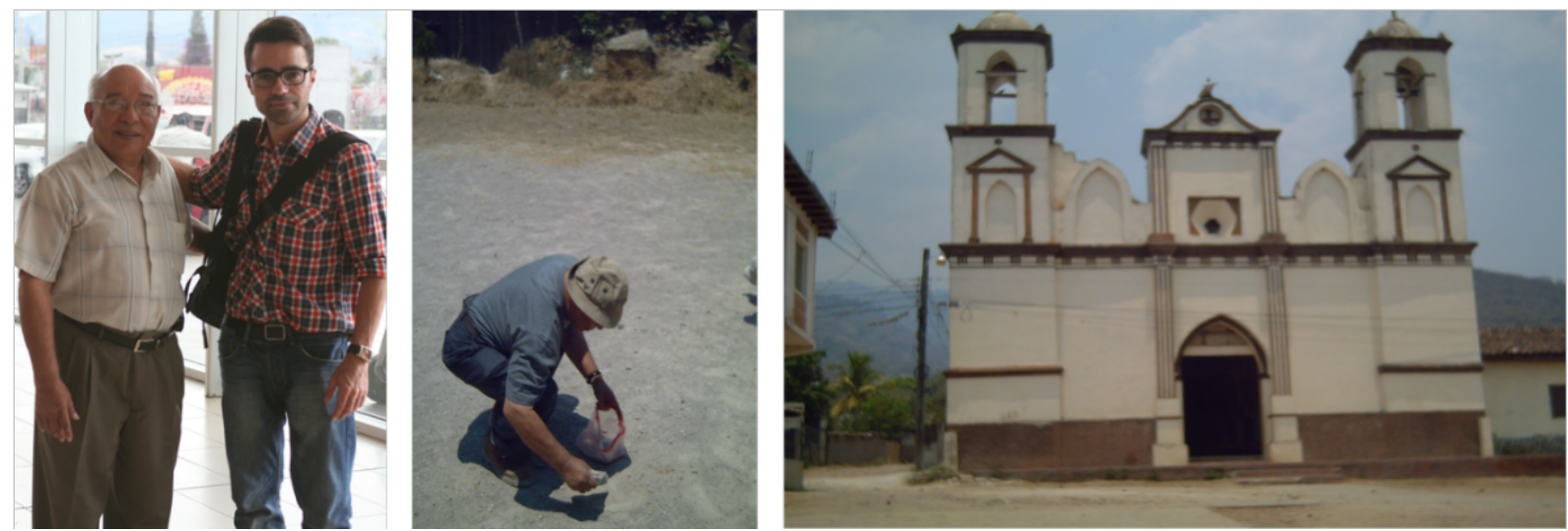

Figura 4: Gira de campo para recolección de muestras en Jesús de Otoro junto al profesor Juan Gregorio Rejas. Créditos: Juan Gregorio Rejas.

"Arqueología de las zonas de estudio de un proyecto de teledetección en la República de Honduras", con los coautores Arnulfo Ramírez, César Rodríguez, Yeny Castellanos, Rafael Corrales y Juan Gregorio Rejas, en la Revista Ciencias Espaciales en 2011.

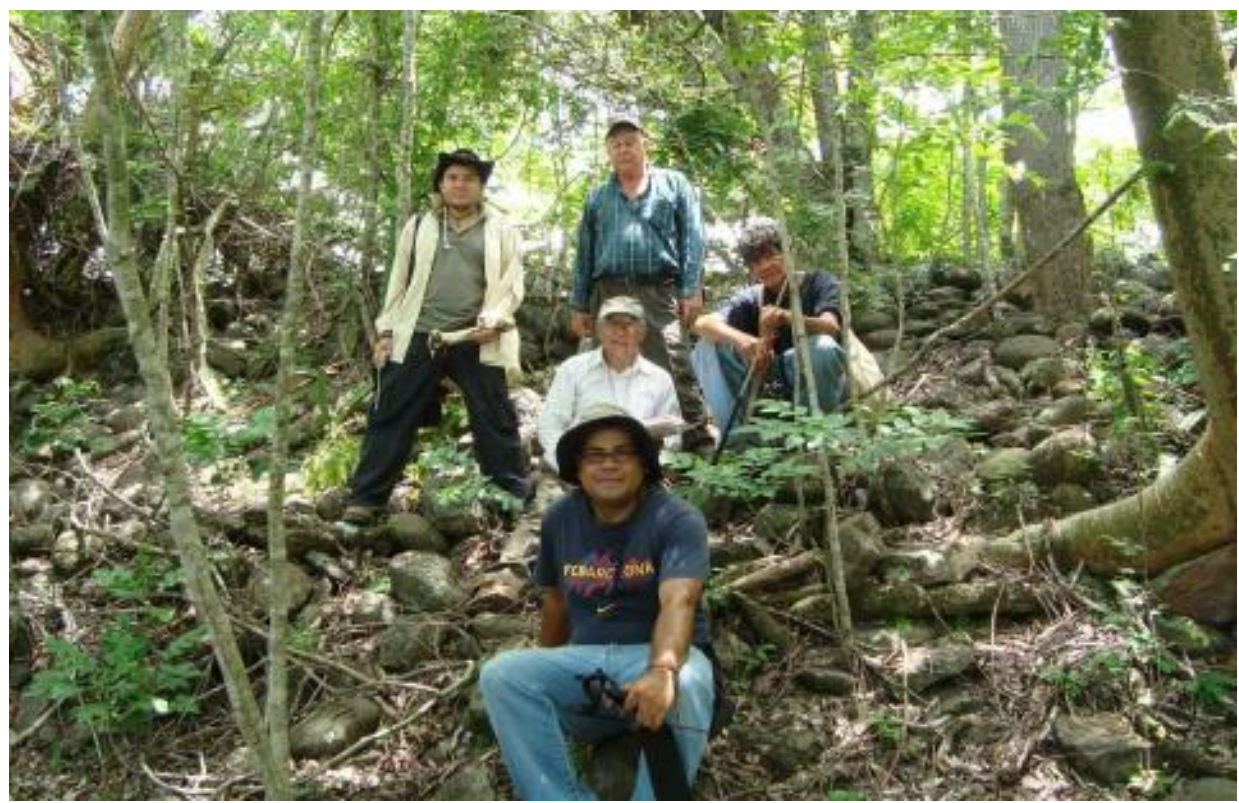

Figura 5: Gira de campo en Jesús de Otoro. De izquierda a derecha, de arriba hacia abajo aparecen: Cesar Rodríguez, Nery Fiallos, David Williams, Vito Veliz y Bertilio Amaya. Créditos: Josué Erubel Ramos.

A partir del trabajo de investigación realizado en la Facultad de Ciencias Espaciales, publicó una serie de artículos, de forma individual o en coautoría con otros investigadores, en las temáticas de arqueología, arqueoastronomía y tecnologías de la información geográfica (tabla 5). 
Tabla 5: Artículos en revistas y temáticas relaciones con la Facultad de Ciencias Espaciales

\begin{tabular}{cl}
\hline No. & Artículos \\
\hline 1 & Fechas Asignadas a la Estela E de Copán. Ciencia y Tecnología, No. 13: 1-10, Universidad \\
& Nacional Autónoma de Honduras, Tegucigalpa, 2003. \\
2 & Base de datos de eventos solares observados desde la Plaza del Sol del Parque Arqueológico \\
de Copán Ruinas. (Coautor). Ciencia y Tecnología, Publicación bianual, Dirección de \\
Investigación Científica, UNAH. Segunda época, No. 4: 20-35, Tegucigalpa. \\
$3 \quad$ Experiencias de Teledetección en Prospección, Caracterización y Protección del \\
Patrimonio Cultural. Casos de Estudio de Honduras (coautores Juan Gregorio Rejas, \\
María Cristina Pineda de Carías y Aitor Bastarrika). Revista Posgrados UNAH, Vol. II, \\
No. 4. Universidad Nacional Autónoma de Honduras, Diciembre 2010. \\
$4 \quad \begin{array}{l}\text { Arqueología de las zonas de estudio de un proyecto de teledetección en la República } \\
\text { de Honduras (coautores Arnulfo Ramírez, César Rodríguez, Yeny Castellanos, }\end{array}$ \\
Rafael Corrales y Juan Gregorio Rejas). Revista Ciencias Espaciales, Vol. 4, No.1 \\
Primavera. Universidad Nacional Autónoma de Honduras, 2011. \\
5 La Arqueoastronomía y la conservación del patrimonio cultural hondureño. Revista \\
Ciencias Espaciales, Vol. 4, No. 2 Otoño, Universidad Nacional Autónoma de \\
Honduras, 2011. \\
El Centro de documentación arqueoastronómica y afines (coautores César \\
Rodríguez, Bertilio Amaya). Revista Ciencias Espaciales, Vol. 4, No. 2 Otoño, \\
Universidad Nacional Autónoma de Honduras, 2011. \\
Detalles sobre la ubicación y la orientación de los altares G del Parque Arqueológico \\
de Copán, Honduras (coautores María Cristina Pineda de Carías, Ricardo Agurcia \\
Fasquelle). Revista Ciencias Espaciales, Vol. 9, Núm. 2 Otoño, Universidad Nacional \\
Autónoma de Honduras, 2016.
\end{tabular}

Fuente: Elaboración propia a partir de la hoja de vida del profesor Vito Veliz y repositorios institucionales.

En 2012, se retira de la Universidad Nacional Autónoma de Honduras, dejando un legado importante en el departamento de Arqueoastronomía y Astronomía Cultural, la Facultad de Ciencias Espaciales y la UNAH, en general.

No obstante, de octubre a diciembre de 2015, el profesor Veliz fue nombrado primer director del Instituto de Arqueoastronomía, Patrimonio Cultural y Natural (IARPACUNA), de la Facultad de Ciencias Espaciales, cuya sede está en Ciudad de Copán Ruinas. Fue contratado para organizar y asumir la dirección del instituto, ante la necesidad de que se sentaran las bases para el funcionamiento del recién creado Instituto. En la figura 6 se observa el acto de juramentación como director.

El primer Congreso de Ciencias Espaciales de noviembre de 2019, fue el último evento académico donde los compañeros de la Facultad de Ciencias Espaciales compartieron con el profesor Vito (figura 7). En ese evento, junto a la profesora María Cristina Pineda de Carías, presentaron una ponencia, donde describieron parte de los hallazgos de la investigación arqueoastronómica en la Plaza Principal de Copán. Esos resultados forman parte de un recorrido investigativo en ese sitio de casi dos décadas. Pero, además, de disfrutar de ese intercambio académico, se compartieron las amenas pláticas durante los descansos entre conferencias, recordando años pasados y los proyectos futuros. 


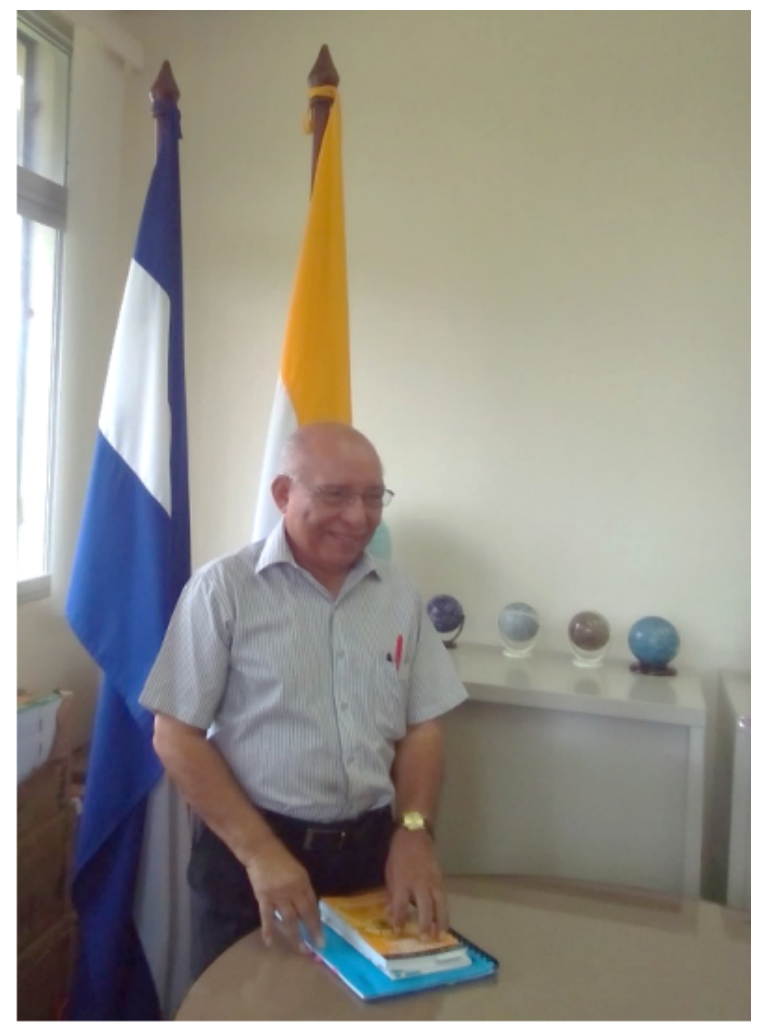

Figura 6: Juramentación como director del Instituto de Arqueoastronomía, Patrimonio Cultural y Natural (IARPACUNA).

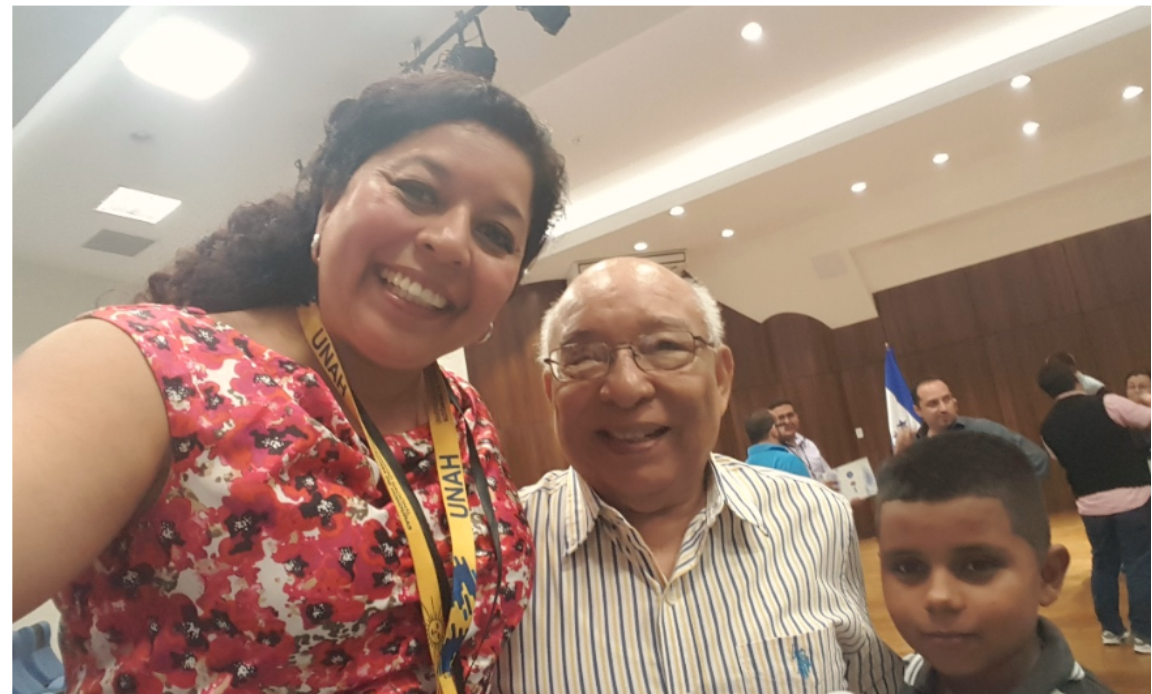

Figura 7: Profesor Vito Veliz, junto a su nieto y la profesora Yeny Castellanos, en el Primer Congreso de Ciencias Espaciales 2019. Créditos: Yeny Castellanos, 2019. 


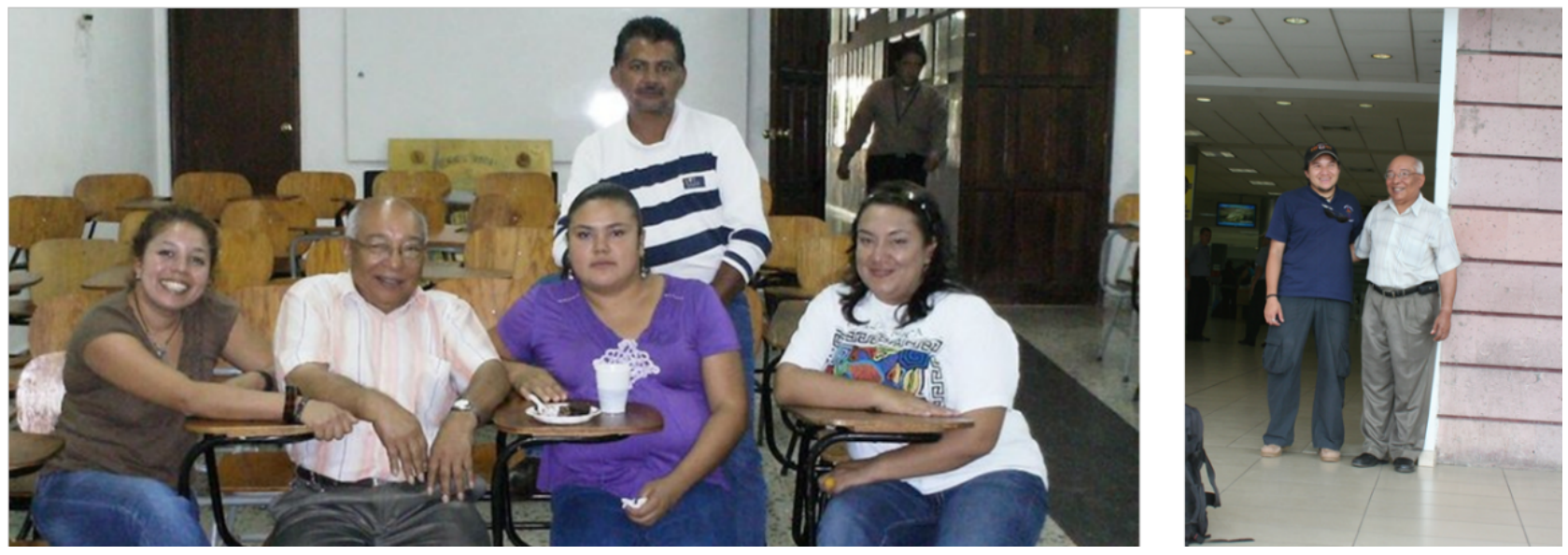

Figura 8: Junto a sus compañeros de trabajo. Foto de la izquierda, orden de izquierda a derecha, de arriba hacia abajo: Laureano Maradiaga, Yeny Castellanos, Vito Veliz, Eva Julia Bonilla y Cristina Argueta; foto de la derecha: Cesar Rodríguez y Vito Veliz.

El profesor Vito es recordado por sus compañeros de trabajo por su humildad, cordialidad y solidaridad. En una anécdota relatada por un compañero de trabajo:

Una anécdota que recuerdo de él está relacionada con su gran calidad humana. Una vez me contó que cuando realizaban estudios para el embalse de El Cajón él notaba que a la hora del almuerzo la gente de la localidad llegaba adonde ellos estaban. Él se preguntaba por qué la gente siempre hacía eso, al inicio le parecía de mala educación que llegaran solo a verlos comer. Después supo que la gente llegaba porque tenían hambre y no tenían que comer. Y llegaban con la esperanza de que ellos les pudieran dar. Hasta se atragantó cuando me contó eso porque me dijo que cuando lo recordaba le daban ganas de llorar. Era un gran ser humano el profe. (B. Amaya, comunicación personal, diciembre del 2020)

En mi caso, yo conocí personalmente al profesor Vito Veliz cuando ingresé a trabajar en el Departamento de Arqueoastronomía y Astronomía Cultural de la UNAH, donde él ejercía la jefatura de esta unidad. Desafortunadamente, el profesor se retiró del trabajo en la Universidad el siguiente año. Sin embargo, siempre mantuvo una relación cercana con el equipo de trabajo del departamento y continúo colaborando en diferentes actividades de la Facultad (figura 8). Se le recordará por su notable trayectoria académica y profesional; su disposición para enseñar a estudiantes, profesores principiantes y compañeros de trabajo; y especialmente por su humildad, cordialidad y solidaridad.

Me quedo y termino con las palabras que culminan uno de sus libros:

Debemos pensar seriamente en los demás. Debemos considerar que muchas de nuestras prácticas (tala de bosques, producción de armas, etc.) son lesivas para nosotros y lo serán mucho más para futuras generaciones. Debemos dar. [...] En otras palabras, debemos amar. Es decir, en todas nuestras relaciones tratar de ser comprensivos, pacientes, compasivos, serviciales, altruistas, futuristas (Véliz, 1988: 85).

\section{Agradecimientos}

Un profundo agradecimiento a Ricardo Agurcia Fasquelle, Juan Gregorio Rejas, Cristina Argueta Canizales, Bertilio Amaya, Josué Erubel Ramos, Cesar Rodríguez y Yeny Castellanos por aportar información e 
imágenes para este artículo.

\section{Referencias}

Agurcia Fasquelle, R. y Véliz, V. (2010). Manual de los Monumentos de Copán, Honduras. Copán: Asociación Copán.

Schöngarth, R., Forrest, D., Milla, E., y Pastrana, R. (2011). Posición de las estelas 10 y 12 respecto del Parque Arqueológico de Copán Ruinas. Revista Ciencias Espaciales, 4(1):87-103.

Véliz, S. V. (1981). Prólogo. Yaxkin, IV(2):77.

Véliz, S. V. (1988). Rudimentos de Antropología. Tegucigalpa: Litografía López. 